\title{
Partial Differential Equations and Function Spaces
}

\author{
Shijun Zheng, ${ }^{1}$ Simone Secchi, ${ }^{2}$ Huoxiong $W u,{ }^{3}$ and Nguyen Cong Phuc ${ }^{4}$ \\ ${ }^{1}$ Department of Mathematical Sciences, Georgia Southern University, Statesboro, GA 30460, USA \\ ${ }^{2}$ Dipartimento di Matematica ed Applicazioni, Università di Milano-Bicocca, 20125 Milano, Italy \\ ${ }^{3}$ School of Mathematical Sciences, Xiamen University, Xiamen 361005, China \\ ${ }^{4}$ Department of Mathematics, Louisiana State University, Baton Rouge, LA 70803, USA
}

Correspondence should be addressed to Shijun Zheng; szheng@georgiasouthern.edu

Received 25 July 2016; Accepted 25 July 2016

Copyright (C) 2016 Shijun Zheng et al. This is an open access article distributed under the Creative Commons Attribution License, which permits unrestricted use, distribution, and reproduction in any medium, provided the original work is properly cited.

It is well known that PDEs and the theory of function spaces have played a central role in the mathematical analysis of problems arising from mathematical physics, biology, and other branches of modern applied sciences. This special issue addresses the current advances in these two broad areas; in particular it focuses on the connections and interactions between them. We are happy that this issue has received the attention of active researchers with interesting and valuable contributions in the field. Topics cover areas from existence and nonexistence theorems for degenerate differential operators to multilinear fractional singular integral operators on weighted function spaces, which might show applications of theory of function spaces in understanding the qualitative as well as quantitative behaviors of PDEs.

This special issue consists of five research papers. L. Dai et al. obtain the existence of solutions to an elliptic equation in a bounded domain for a Leray-Lions operator acting from a weighted Sobolev space into its dual. The paper by Q. Wu et al. establishes the nonexistence of solutions to a semilinear elliptic equation with unbounded inhomogeneous power nonlinearity. They show that there exists no solution with finite Morse index in the energy-subcritical regime. This is a Liouville type theorem that has proven to be a powerful tool in obtaining a priori bound for solutions of elliptic equations. The paper by J. Cunanan and Y. Tsutsui deals with trace operators of Wiener amalgam spaces using frequency uniform decomposition operators and maximal inequalities. Wiener amalgam spaces, together with modulation spaces, were introduced in the 1980s and are now widely used for various problems in PDE and harmonic analysis.
They resemble Triebel-Lizorkin spaces in the sense that one takes $L^{p}\left(\ell^{q}\right)$ norms using frequency uniform decomposition operators through unit cubes instead of dyadic annuli. S. He and $\mathrm{X}$. Tao study the boundedness of multilinear fractional integral operators as well as the boundedness of multilinear maximal singular integral operators with rough kernels on weighted Morrey spaces. Y. Xie et al. obtain the existence and the structure of a compact uniform attractor for a nonautonomous diffusion equation with fading memory, by verifying the uniform asymptotic compactness of a family of processes using asymptotic contractive method. These results are proven under the conditions that the nonlinearity satisfies the polynomial growth of arbitrary order and the time dependent forcing term is only translation-bounded.

We hope that the readers who are interested in analysis and applications of function spaces, differential operators, and PDEs will find useful information and perspectives in this special issue. Meanwhile, we also hope that in the near future we can see new papers be published based on this issue.

\section{Acknowledgments}

The editors thank all the contributors and reviewers who have spent valuable time during the peer-review process. Without the significant contributions made by these researchers, the publication of the special issue would not be possible.

Shijun Zheng

Simone Secchi

Huoxiong Wu

Nguyen Cong Phuc 


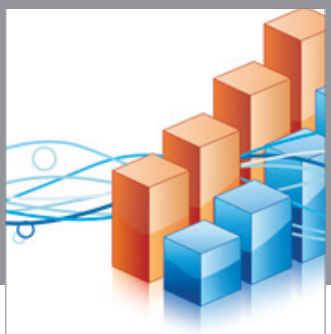

Advances in

Operations Research

vatem alat4

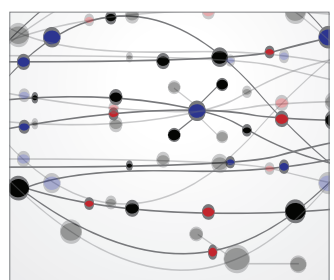

\section{The Scientific} World Journal
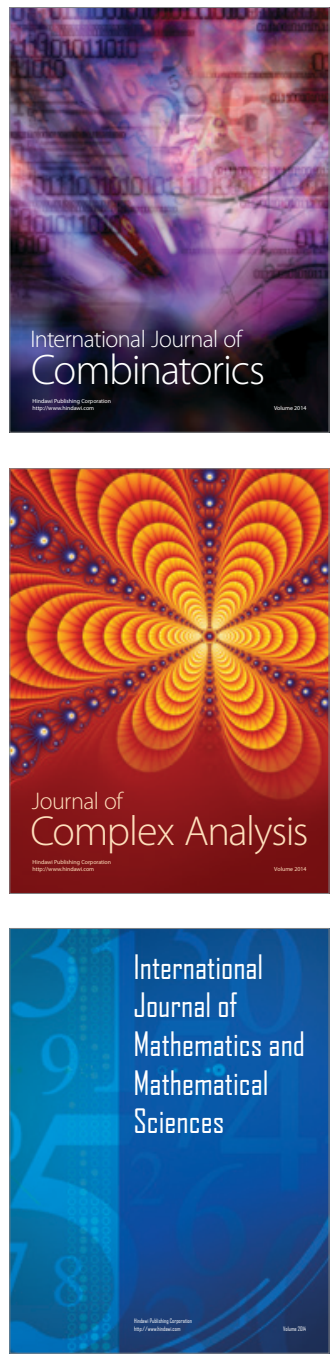
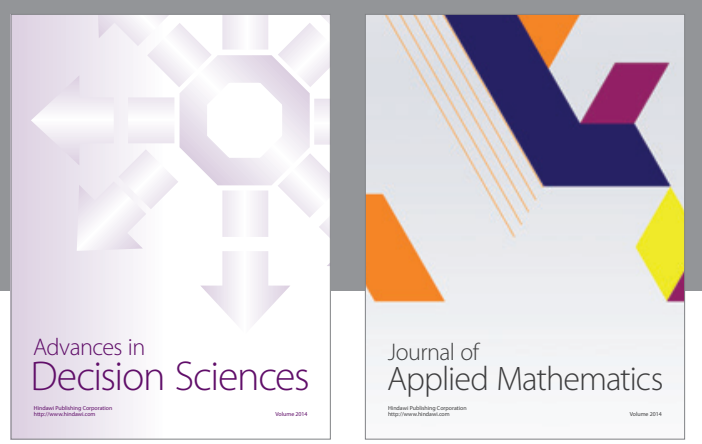

Algebra

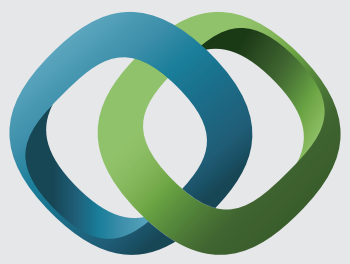

\section{Hindawi}

Submit your manuscripts at

http://www.hindawi.com
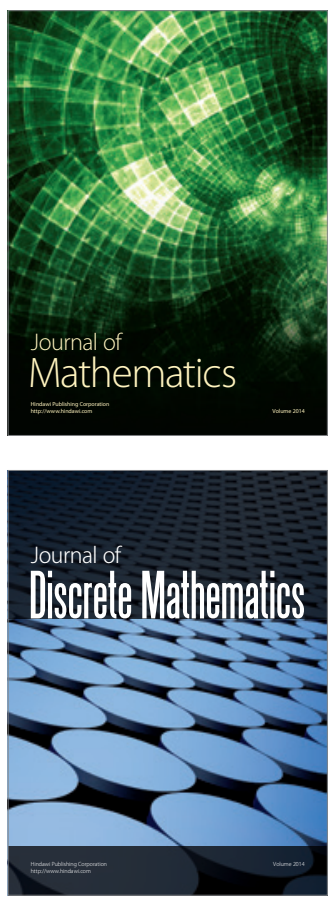

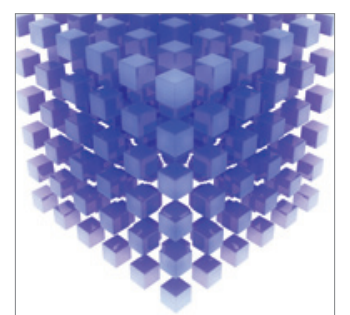

Mathematical Problems in Engineering
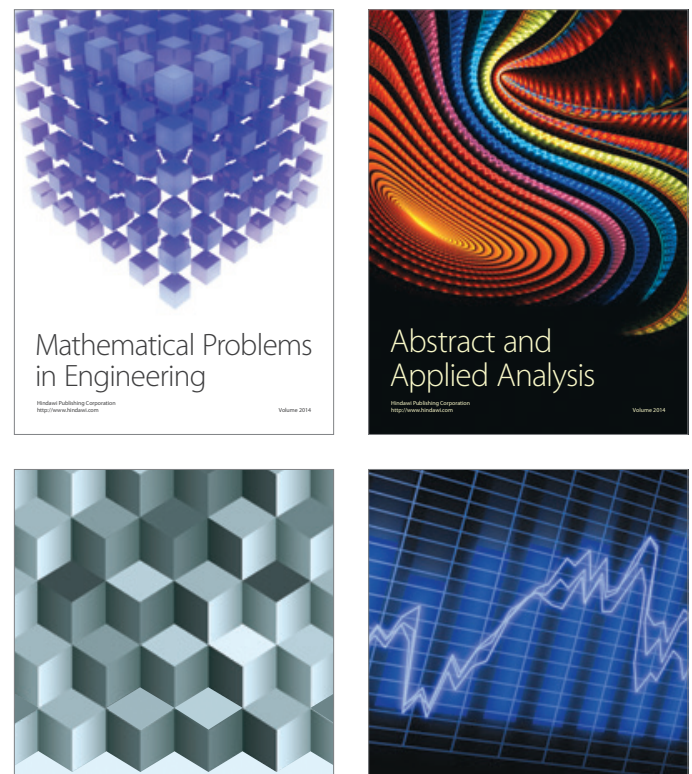

Journal of

Function Spaces

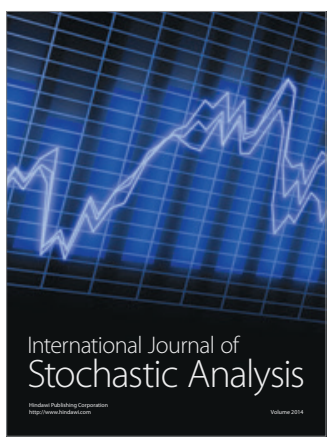

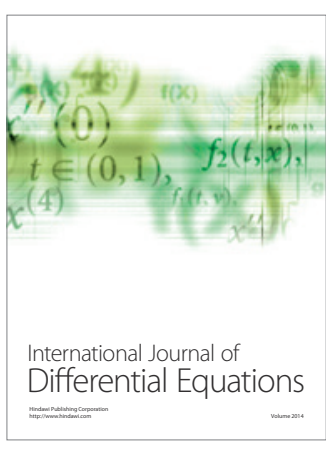
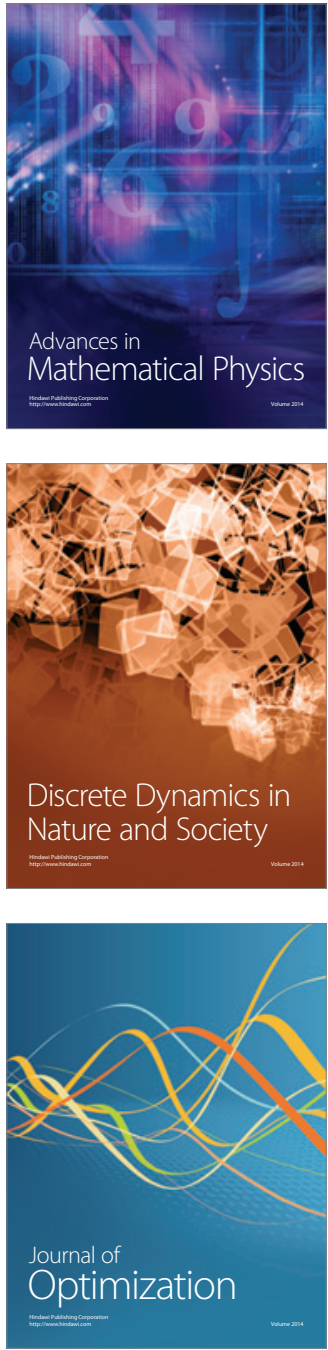